\title{
Ups and Downs: Team Performance in Best-of-Seven Playoff Series
}

\author{
Tim B. Swartz, Aruni Tennakoon, Farouk Nathoo, \\ Parminder S. Sarohia and Min Tsao *
}

\begin{abstract}
This paper explores the impact of the status of a playoff series on team performance in a best-of-seven playoff format. Betting line data are collected on more than 1200 playoff matches from the National Basketball Association (NBA) and the National Hockey League (NHL) from 2003 through 2011. Regression methodology is used to suggest that teams in desperate situations (i.e. those teams close to elimination in a series) tend to have better results than when they are not in desperate situations. However, there also seems to exist situations where the mountain is too steep to climb and desperation leads to capitulation. In comparing the two leagues, it appears that the effects due to the status of a series are less prominent in the NHL than in the NBA.
\end{abstract}

Keywords : home team advantage, NBA, NHL, regression, sportsbook betting.

${ }^{*}$ Tim Swartz is Professor and Aruni Tennakoon is an MSc graduate, Department of Statistics and Actuarial Science, Simon Fraser University, 8888 University Drive, Burnaby BC, Canada V5A1S6. Min Tsao is Professor, Farouk Nathoo is Associate Professor and Parminder Sarohia is an MSc graduate, Department of Mathematics and Statistics, University of Victoria, Victoria BC, Canada V8W3P4. Swartz, Nathoo and Tsao have been partially supported by the Natural Sciences and Engineering Research Council of Canada. The authors are appreciative of the comments provided by an anonymous referee. The comments led to an improvement in the manuscript. 


\section{INTRODUCTION}

Its the playoffs of the National Basketball Association (NBA) and your favourite team is facing elimination as they are down 3-0 in a best-of-seven playoff series. How will they respond in the next game? Will they feel discouraged and give up? Will they play a typical game? Or will they dig deep and give it their ultimate effort?

Interestingly, there are varied opinions on how teams play given the results of previous games. Many quantitative analyses of sporting events begin with the assumption of independent and identically distributed (iid) Bernoulli trials. Tversky and Gilovich (1989) support this assumption in the context of free throws in basketball as they argue against the existence of the "hot hand". However, it is clear that the iid assumption is invalid in many situations where conditions change. For example, in considering successive games in a playoff series, outcome probabilities change according to injuries and the home team advantage (Stefani 2008). Schembri, Bedford, O'Bree and Park (2011) suggest that there are incentives to win and lose games in the NBA with respect to qualifying for the playoffs and in improving the probability of attaining a high draft pick. Stern (1998a) also criticizes the iid assumption using game results from the NBA and NHL (National Hockey League) playoffs. In addressing the independence assumption, Stern (1998a) suggests that there is evidence of independence in the NHL playoffs, but in the NBA, teams may be more likely to lose once they are leading in a series. On the other hand, Jackson (1993) argues for the "success breeds success" phenomenom in tennis.

Our investigation of the effects due to the status of a series is nonstandard in that we do not analyze the scoring outcomes of games. Instead, we analyze betting lines as provided by sportsbooks. We prefer betting lines for the simple reason that they are less variable than the scores of sporting matches, and hence provide us with more information. For example, in the NBA, it is common for a team to win by more than 10 points against an evenly matched opponent. However, if one were to look at closing NBA pointspreads offered 
by various sportsbooks, it would be very unlikely to find a pointspread that differs from another pointspread by more than two points.

It is also important to realize that betting lines reflect reality and therefore accurately describe the stochastic nature of games. In market language, we say that sportsbooks are efficient in the sense that there does not exist a long-term strategy to exploit the sportsbook. The efficiency of markets (both financial and sports betting) has wide support in the literature (Gandar, Brown, Dare and Zuber 1998, Sauer 1998 and Stern 1998b). A simple argument for the efficiency of sports betting markets is the continuation of sports gambling as seen in Las Vegas. If the Las Vegas sports betting markets were inefficient, the betting advantage would put the industry out of business.

In section 2, we provide a review of various elements of sports gambling that is relevant to our data. In particular, we convert sportsbook betting lines from the NBA and the NHL into variables measuring team strength at a hypothetical neutral site. In section 3, we introduce two models that are used to analyze the effects due to the status of a series in terms of team strength. The models are then fit to NBA playoff data and NHL playoff data. We conclude with a short discussion in section 4 .

The material presented here grew out of the work of the MSc thesis of Sarohia (2010) at the University of Victoria and the MSc project of Tennakoon (2011) at Simon Fraser University. Whereas both approaches yielded qualitatively similar results, the work of Sarohia (2010) was based on the analysis of actual scoring data whereas Tennakoon (2011) considered data from betting lines.

\section{REVIEW OF SPORTS BETTING LINES}

Our analysis uses sportsbook betting lines to measure the amount that one team is favoured

over another team. The website www.covers.com provides historical lines for NBA playoff games beginning in 2003 and NHL playoff games beginning in 2006. 
For illustration, consider the first game of the 2008 NBA finals between the Los Angeles Lakers and the Boston Celtics which took place in Boston on June 5, 2008. The betting line corresponding to the pointspread was given by

$$
\begin{array}{lll}
\text { Los Angeles } & +3 & -110 \\
\text { Boston } & -3 & -110
\end{array}
$$

An interpretation of the betting line (1) is that a gambler may bet $\$ 110$ on Boston. Should Boston win by more than three points (the pointspread), the $\$ 110$ is returned along with a profit of $\$ 100$. If Boston wins by exactly three points, this is referred to as a push and the $\$ 110$ is returned. If Boston wins by one or two points, or loses the game, then the gambler loses the $\$ 110$ wager. Conversely, suppose that a gambler bets $\$ 110$ on Los Angeles. Then the $\$ 100$ is returned along with a profit of $\$ 110$ if Los Angeles wins or if Boston wins by one or two points. The wager of $\$ 110$ is returned if a push occurs and the gambler loses the $\$ 110$ wager if Boston wins by more than three points.

Note that there are variations of the situation described above. A gambler does not need to bet $\$ 110$, but may bet any amount not exceeding the limit imposed by the sportsbook, and the amount won/lost is then proportional to the amount wagered. Also, the pointspread does not need to be in an integer. Sometimes it can be a number such as 2.5 in which case there is no possibility of a push.

Most importantly, we note that the odds -110 (expressed as American odds) are not constant from match to match, and the odds determine the vigorish for the sportsbook. Suppose that the betting line is posted as in (1), and that we have two gamblers where one gambler wagers $\$ 110$ on Los Angeles and the other gambler wagers $\$ 110$ on Boston. Ignoring the possibility of a push, the sportsbook collects $\$ 220$ but pays out only $\$ 210$. In this case, the sportsbook has made a profit of $\$ 10$, and profits allow sportsbooks to operate. Therefore, the "safe" strategy for a sportsbook is to attempt to set pointspreads so as to balance the amount bet on each team. American odds of -110 are known as a dime line but sportsbooks are known to offer both more attractive and less attractive odds. For example, 
a gambler would prefer to wager at American odds of -105 than the common dime line. A sportsbook offering odds at -105 needs to be more confident in the efficiency of their odds and they need a large gambling clientele.

For the NBA, we wish to convert betting lines involving pointspreads to variables denoting team strength on a hypothetical neutral court. Let $\mu$ denote the pointspread for a team of interest where negative values indicate that the team is favoured to win. Stern and Mock (1998) suggest that the point differential by which the team of interest defeats its opponent in US college basketball is well approximated by the normal $\left(-\mu, \sigma^{2}\right)$ distribution. Likewise, we use the normal assumption in the context of the NBA. Although there is some support (Gandar, Zuber and Lamb 2001) for an NBA regular season home court advantage of $h \approx 4.0$ points, we choose $h=3.4$. Our rationale is based on the lack of balance in the regular season in the sense that visiting teams more often play back-to-back games. In the playoffs, both teams are equally rested. Our choice $h=3.4$ is consistent with Entine and Small (2008) who analyzed the 2004-2005 and 2005-2006 NBA seasons, and concluded that the number of points $h$ corresponding to the home court advantage for equally rested teams has a confidence interval of $(2.46,3.40)$. For the standard deviation, we adopt the estimate $\sigma=11.1$ provided by Entine and Small (2008). Alternative but comparable values of $\sigma$ have also been reported in the literature. For example, an analysis by Gibbs (2007) using NBA data over the period 1993-2007 suggests $\sigma=11.4$. Larsen, Price and Wolfers (2008) fit a normal distribution where $\sigma=11.6$ is obtained (personal communication). In any case, our results in section 3.1 are not sensitive to small changes in the home court advantage parameter $h$ and the standard deviation parameter $\sigma$.

Therefore, as a measure of strength for an NBA team of interest with pointspread $\mu$, we define

$$
y=\ln (p /(1-p))
$$


where

$$
p= \begin{cases}\Phi((-\mu-h) / \sigma) & \text { home game for the team of interest } \\ \Phi((-\mu+h) / \sigma) & \text { away game for the team of interest }\end{cases}
$$

is the probability of victory for the team of interest on a hypothetical neutral court and $\Phi$ is the distribution function of the standard normal distribution. Note that the variable $y$ in (2) has been transformed to the real line. Positive (negative) values of $y$ indicate that the team of interest is stronger (weaker) than its opponent.

For the NHL playoff data, a normal distribution for goal differentials is clearly inappropriate due to the paucity of goals scored in games. Alternatively, instead of a betting line corresponding to the pointspread, our data takes the form of a moneyline. For illustration, consider the first game of the 2008 NHL finals between the Detroit Red Wings and the Pittsburgh Penguins which took place in Detroit on May 24, 2008. The moneyline was given by

$$
\begin{array}{ll}
\text { Pittsburgh } & +130 \\
\text { Detroit } & -140
\end{array}
$$

The interpretation of the moneyline (4) corresponds directly to whether a team wins or loses. For example, suppose that a gambler wagers $\$ 140$ on Detroit. Then $\$ 140$ is returned along with a profit of $\$ 100$ if Detroit wins, and the gambler loses the $\$ 140$ wager if Detroit loses. On the other hand, consider a gambler who wagers $\$ 100$ on Pittsburgh, and note that the American odds of +130 has a positive sign. In this case, the gambler receives $\$ 100$ back along with a profit of $\$ 130$ if Pittsburgh wins, and the gambler loses the $\$ 100$ wager if Pittsburgh loses. In (4), Detroit is the favourite and the American odds of +130 is provided to lure wagers towards Pittsburgh.

For the NHL, we wish to convert moneyline data to win probabilities on hypothetical neutral ice. Ignoring the sign in (4), the difference between +130 and -140 represents the vigorish. If there were no vigorish, then one might see a moneyline such as Detroit -135 and 
Pittsburgh +135 , in which case there would be no systematic advantage for the sportsbook and 135 would represent the "true" moneyline. To convert the moneyline data to win probabilities, we eliminate the vigorish by taking the "midpoint" between the odds. For example, in (4), we let $p$ be the probability that Detroit wins, and we set the expected profit from a $\$ 135$ wager on Detroit equal to zero;

$$
0=100 p-135(1-p)
$$

which gives $p=0.574$. Note that we obtain the same result by considering a wager on Pittsburgh and setting the expected profit equal to zero.

For the NHL data, our final step in determining a team strength variable corresponding to hypothetical neutral ice requires the incorporation of the home ice advantage. Over the last five regular seasons of the NHL, 2006-2007 through 2010-2011, the home team has won 3353 times in 6150 games played. We therefore estimate the home ice winning percentage as

$$
\frac{3353}{6150} \rightarrow 54.5 \%
$$

which is a much smaller effect than in the NBA where the home team wins $60.5 \%$ of its games (Stefani 2008). To adjust to the case of hypothetical neutral ice, we then take the calculated winning probability $p$ above, and subtract 0.045 if the team of interest is playing at home, and add 0.045 if the team is away. In the context of the above example, Detroit is the home team and therefore its winning percentage on hypothetical neutral ice is $0.574-0.045=$ 0.529. And using (2), our measure of strength for Detroit on hypothetical neutral ice is $y=\ln (0.529 / 0.471)=0.116$.

\section{STATISTICAL MODELS}

Consider a best-of-seven playoff series following the sequence $H H A A H A H$ where $H$ denotes a home game and $A$ denotes an away game. We refer to the team which plays the first game 
at home as the reference team, and typically, the reference team is the higher seeded team and the favoured team to win the playoff series.

In a best-of-seven playoff series following the sequence $H H A A H A H$, we denote the state of the series as $w-l$ where the reference team has won $w$ games and has lost $l$ games. We note that there are 16 possible states which are listed in table 1.

\begin{tabular}{cc||cc||cc||cc} 
State & $w-l$ & State & $w-l$ & State & $w-l$ & State & $w-l$ \\
\hline 0 & $0-0$ & 4 & $0-1$ & 8 & $0-2$ & 12 & $0-3$ \\
1 & $1-0$ & 5 & $1-1$ & 9 & $1-2$ & 13 & $1-3$ \\
2 & $2-0$ & 6 & $2-1$ & 10 & $2-2$ & 14 & $2-3$ \\
3 & $3-0$ & 7 & $3-1$ & 11 & $3-2$ & 15 & $3-3$
\end{tabular}

Table 1: The 16 possible states $w-l$ in a best-of-seven playoff series with respect to the reference team.

In this paper, the scientific question which we pose is the following, "Does the reference team play differently according to the state of the playoff series?" For example, does the reference team play better in a 1-3 series facing elimination than in a 3-1 series where they are comfortably ahead? With the home team advantage prominent in sport, an analysis should take this factor into account. An analysis also needs to take into account the relative form (i.e. inherent ability) of the competing teams. We want to separate these two factors from the impact due to the state of the series.

To initiate model development, consider the $i$ th playoff series. We let $y_{i, j}$ denote the strength of the reference team corresponding to state $j$ at a hypothetical neutral site as developed in section 2. Note that whereas $y_{i, 0}$ is always observed, we do not have observations $y_{i, j}$ for all states $j=1, \ldots, 15$. We propose that the strength of the reference team $y_{i, j}$ is a combination of its form relative to its opponent and the effect due to the state of the playoff series. We therefore tentatively consider the model

$$
y_{i, j}=f_{i, j}+s_{j}+\epsilon_{i, j}
$$


where in series $i, f_{i, j}$ is the relative form of the reference team corresponding to state $\mathrm{j}, s_{j}$ is the psychological effect corresponding to state $j$, and $\epsilon_{i, j}$ is the error term. Note that our use of the term "psychological effect" is actually the remaining systematic effect with respect to the state of a series after form and home advantage have been removed. It is difficult for us to imagine that such an effect could be due to anything other than mental factors.

A problem with model (5) is that it is not identifiable. We cannot separate the form term $f_{i, j}$ from the psychological component $s_{j}$. To deal with the issue, we hypothesize that the reference team's form is determined in the first match of the series, and then changes only slightly throughout the series. This is clearly an approximation to reality as a variety of issues are relevant to form including injuries, strategic adjustments, etc. In defining Model A, we modify (5) according to the constant form assumption

$$
f_{i, j}=f_{i, 0}
$$

from which

$$
\begin{aligned}
y_{i, j}-y_{i, 0} & =\left(f_{i, 0}+s_{j}+\epsilon_{i, j}\right)-\left(f_{i, 0}+s_{0}+\epsilon_{i, 0}\right) \\
& =\left(s_{j}-s_{0}\right)+\left(\epsilon_{i, j}-\epsilon_{i, 0}\right) .
\end{aligned}
$$

Reparametrizing, Model A can be expressed as

$$
y_{i, j}-y_{i, 0}=d_{j}+\epsilon_{i, j}^{*}
$$

where $d_{j}=s_{j}-s_{0}$ represents the change in the psychological effect from the beginning of the series to state $j$, and $\epsilon_{i, j}^{*}$ is the error term. Therefore, by taking the difference $y_{i, j}-y_{i, 0}$ as the dependent variable, we have eliminated the form component from the right side of the regression equation (6).

Alternatively, one may conjecture that current form is better represented by form established prior to the previous match which is modified by the result of the previous match. In other words, we modify (5) according to

$$
f_{i, j}=f_{i, \mathrm{prev}}+\delta\left(2 I_{w}-1\right)
$$


where we use the subscript "prev" to indicate the state that preceded the current state $j$, and $I_{w}$ is an indicator variable where $I_{w}=1(0)$ implies that the reference team won (lost) the previous match. Therefore the current form is the previous form updated by $\delta$ corresponding to a win and $-\delta$ corresponding to a loss in the previous game. This leads to the model

$$
\begin{aligned}
y_{i, j}-y_{i, \mathrm{prev}} & =\left(f_{i, \mathrm{prev}}+\delta\left(2 I_{w}-1\right)+s_{j}+\epsilon_{i, j}\right)-\left(f_{i, \mathrm{prev}}+s_{\mathrm{prev}}+\epsilon_{i, \mathrm{prev}}\right) \\
& =\left(s_{j}-s_{\mathrm{prev}}\right)+\delta\left(2 I_{w}-1\right)+\left(\epsilon_{i, j}-\epsilon_{i, \mathrm{prev}}\right) .
\end{aligned}
$$

Reparametrizing, Model B can be expressed as

$$
y_{i, j}-y_{i, \mathrm{prev}}=d_{j, \mathrm{prev}}+\delta\left(2 I_{w}-1\right)+\epsilon_{i, j}^{*}
$$

where $d_{j, \text { prev }}=s_{j}-s_{\text {prev }}$ represents the change in the psychological effect from the previous state to state $j$, and $\epsilon_{i, j}^{*}$ is the error term.

In developing regression code for Model B, we need to be careful with the parametrization in (7). In table 2 , we list the 24 possibilities corresponding to $d_{j \text {,prev }}=s_{j}-s_{\text {prev }}$, and we indicate the simplified parametrization in terms of the 15 variables $d_{1}, \ldots, d_{15}$ originally defined in (6). The common parametrization facilitates a direct comparison between Model A and Model B.

\begin{tabular}{ll||ll||ll}
$d_{j, \mathrm{prev}}$ & Coding & $d_{j, \mathrm{prev}}$ & Coding & $d_{j, \mathrm{prev}}$ & Coding \\
\hline$d_{1,0}$ & $d_{1}$ & $d_{7,3}$ & $d_{7}-d_{3}$ & $d_{11,10}$ & $d_{11}-d_{10}$ \\
$d_{2,1}$ & $d_{2}-d_{1}$ & $d_{7,6}$ & $d_{7}-d_{6}$ & $d_{12,8}$ & $d_{12}-d_{8}$ \\
$d_{3,2}$ & $d_{3}-d_{2}$ & $d_{8,4}$ & $d_{8}-d_{4}$ & $d_{13,9}$ & $d_{13}-d_{9}$ \\
$d_{4,0}$ & $d_{4}$ & $d_{9,5}$ & $d_{9}-d_{5}$ & $d_{13,12}$ & $d_{13}-d_{12}$ \\
$d_{5,1}$ & $d_{5}-d_{1}$ & $d_{9,8}$ & $d_{9}-d_{8}$ & $d_{14,10}$ & $d_{14}-d_{10}$ \\
$d_{5,4}$ & $d_{5}-d_{4}$ & $d_{10,6}$ & $d_{10}-d_{6}$ & $d_{14,13}$ & $d_{14}-d_{13}$ \\
$d_{6,2}$ & $d_{6}-d_{2}$ & $d_{10,9}$ & $d_{10}-d_{9}$ & $d_{15,11}$ & $d_{15}-d_{11}$ \\
$d_{6,5}$ & $d_{6}-d_{5}$ & $d_{11,7}$ & $d_{11}-d_{7}$ & $d_{15,14}$ & $d_{15}-d_{14}$
\end{tabular}

Table 2: The 24 parameters $d_{j, \text { prev }}$ in Model B and their simplified parametrization in terms of $d_{1}, \ldots, d_{15}$. 


\subsection{NBA Data Analysis}

We have collected data on 9 years of NBA playoffs, from 2003 through 2011. During this period, each playoff series followed a best-of-seven $H H A A H A H$ format except for the final championship series in each year which followed a $H H A A A H H$ format. With 16 eligible teams in the playoffs each year, this leads to 14 yearly playoff series following the HHAAHAH format. Our data therefore consists of $9(14)=126$ series comprising 704 matches. Whereas the dataset is large, there are some states which are relatively rare (e.g. State 12 has only four observations).

In table 3, we provide the results from fitting Model A and Model B to the NBA data. Our first observation is that according the $R^{2}$ fit diagnostic, Model A does not fit as well as Model B. This may have been anticipated since it is reasonable to expect that current form is better approximated by recent form than by initial form in a series. Also, Model B contains the additional parameter $\delta$. In Model B, we observe the seemingly counterintuitive result that $\delta$ is negative. Recall that $\delta$ was intended as an update on form, where a win in the previous match by the reference team was suppose to increase one's assessment of form. Instead, it appears that a team that wins its previous match has a tendency to "relax" and not play as well in the next game. This corroborates the conclusions expressed by Stern (1998a). Consequently, the $\delta$ term is not really a form parameter but is also a psychological parameter.

What is also interesting about table 3 is some of the moderate discrepancies in the estimates between Models A and B, and the fact that the standard errors are nearly identical. For example, we note that $d_{2}, d_{3}, d_{6}, d_{8}$ and $d_{15}$ differ significantly between Models A and B. Our interpretation of this observation is that the estimates from Model A are less reliable. Our rationale is that the assumption of constant form throughout a series is simply not realistic. In analysing the effects (as is done later), we find that plausible explanations exist for the $d_{i}$ in Model $\mathrm{B}$ whereas the task of providing interpretations is more difficult for some of the $d_{i}$ in Model A. For example, in Model A, $d_{8}=-0.16$ suggests that the either the 
reference team is only mildly discouraged having fallen behind 0-2 in a series or that the opponent is only mildly invigorated. Our intuition is that State 0-2 is a devastating position for the reference team and that $d_{8}=-0.41$ provided by Model B is a more realistic estimate.

\begin{tabular}{c||r|r} 
Parameter & Model A & Model B \\
\hline$d_{1}$ & $-0.10(0.03)$ & $-0.05(0.04)$ \\
$d_{2}$ & $-0.26(0.04)$ & $-0.10(0.04)$ \\
$d_{3}$ & $0.21(0.06)$ & $0.45(0.06)$ \\
$d_{4}$ & $0.12(0.05)$ & $0.07(0.05)$ \\
$d_{5}$ & $-0.12(0.04)$ & $-0.15(0.04)$ \\
$d_{6}$ & $-0.05(0.04)$ & $0.18(0.04)$ \\
$d_{7}$ & $0.14(0.05)$ & $0.20(0.05)$ \\
$d_{8}$ & $-0.16(0.12)$ & $-0.41(0.12)$ \\
$d_{9}$ & $-0.07(0.06)$ & $0.03(0.06)$ \\
$d_{10}$ & $0.06(0.04)$ & $0.07(0.04)$ \\
$d_{11}$ & $-0.15(0.04)$ & $-0.20(0.04)$ \\
$d_{12}$ & $-0.32(0.15)$ & $-0.29(0.15)$ \\
$d_{13}$ & $0.00(0.07)$ & $0.12(0.07)$ \\
$d_{14}$ & $-0.31(0.06)$ & $-0.30(0.06)$ \\
$d_{15}$ & $-0.02(0.06)$ & $0.17(0.06)$ \\
$\delta$ & & $-0.05(0.02)$ \\
\hline$R^{2}$ & 0.20 & 0.31
\end{tabular}

Table 3: Parameter estimates, standard errors and diagnostics obtained from fitting Model A and Model B to the NBA data.

Our next task is to arrive at some qualitative understanding of the estimates obtained in Model B. We have been unable to provide a mathematical theory underlying the estimates. For example, one might consider a theory that the psychological impact on the reference team is equivalent under all states where the reference team is a fixed number of games ahead (behind) in the series. Instead, we provide some plausible explanations for the estimates. In table 3, although 8 of the 15 effects for Model B are statistically significant at the 0.01 level of significance, it is really the size of the effect which is meaningful. Consequently, we only discuss cases where $\left|d_{j}\right|>0.2$. Recall that $d_{j}$ is the effect of the $j$ th state relative to the 
beginning of the series on a log-odds scale. Thus $d_{j}>0.2$ corresponds to a 0.05 increase in probability from $p=0.5$. We begin our discussion of the states having a large positive effect on the reference team.

State 3 (3-0): Here the reference team has a commanding lead in the series with only one more win needed over four potential games. The confidence of the reference team and the loss of hope by its opponent provides a huge psychological boost to the reference team. In fact, to date, in the history of the NBA, no team has ever overcome a 3-0 series deficit to win the series (0 out of 99 attempts).

State 7 (3-1): This is an important close-out game for the reference team. Should they win, the series is over. However, if they lose, they face the prospect of an away game six where the opponent is encouraged. Winning in State 7 terminates the series whereas losing gets you perilously close to an all-or-nothing game seven.

State 15 (3-3): This state does not quite satisfy the criterion $d_{j}>0.2$. Although it may appear that both teams have an equal incentive to win, there is a general feeling that NBA home teams are dominant in matches where the series is tied 3-3. In the 30 series from 1999 through 2011 that have been extended to a full seven games, the home team has won $23(77 \%)$ of the matches.

We now discuss states with large negative effects (i.e. $d_{j}<-0.2$ ).

State 8 (0-2) and State 12 (0-3): Analogous to State 3, these are capitulation states where it seems that there is no hope for the reference team. In State 8, the reference team has lost both home games and is facing sure defeat as it goes on the road for the next two games. The prospects are even bleaker for the reference team in State 12.

State 11 (3-2) and State 14 (2-3): These are both situations where the reference team is on the road in the sixth game of the series. In State 11, the reference team may relax, 
having confidence that it can win the series in a game seven environment as suggested by State 15. In State 14, the opposite sort of psychology takes place; the opponent is desperate to win the series at home as it knows that a game seven is likely fatal.

\subsection{NHL Data Analysis}

We have collected data on 6 years of NHL playoffs, from 2006 through 2011. During this period, each playoff series followed a best-of-seven $H H A A H A H$ format. With 16 eligibile teams in the playoffs, this leads to 15 yearly playoff series. Our data therefore consists of $6(15)=90$ series comprising 514 matches.

In table 4, we provide the results from fitting Model A and Model B to the NHL data. As with the NBA data, Model A does not fit as well as Model B. In Model B, we observe that $\delta \approx 0$ which suggests that a win by the reference team is not a clear indicator of improved form. As before, we restrict our discussion to the interpretation of large effects (i.e. cases where $\left.\left|d_{j}\right|>0.2\right)$. Accordingly, State 3 (3-0) and State 7 (3-1) have large positive effects for the reference team with the same interpretation as provided in the NBA study. Additionally, State $10(2-2)$ is also beneficial to the reference team. We justify this effect by emphasizing the importance of the state to the reference team as they do not want to go on the road for game six in a 2-3 deficit. Conversely, State 8 (0-2), State 12 (0-3) and State 14 (2-3) all have negative effects to the reference team for the same reasons provided in the NBA analysis. In addition, State $2(2-0)$ is a negative state for the reference team; they have taken care of business in their first two home games and perhaps they tend to relax when they go on the road. Conversely, the opponent is desperate to win.

\section{DISCUSSION}

How do we reconcile the analyses for the NBA and the NHL? One would think that there are common underlying psychological factors in both leagues with respect to Model B. To 


\begin{tabular}{c||r|r} 
Parameter & Model A & Model B \\
\hline$d_{1}$ & $0.01(0.02)$ & $0.00(0.02)$ \\
$d_{2}$ & $-0.22(0.02)$ & $-0.26(0.02)$ \\
$d_{3}$ & $0.11(0.03)$ & $0.26(0.03)$ \\
$d_{4}$ & $0.09(0.03)$ & $0.10(0.03)$ \\
$d_{5}$ & $-0.16(0.02)$ & $-0.19(0.02)$ \\
$d_{6}$ & $-0.16(0.02)$ & $0.04(0.02)$ \\
$d_{7}$ & $0.14(0.03)$ & $0.22(0.03)$ \\
$d_{8}$ & $-0.14(0.04)$ & $-0.22(0.04)$ \\
$d_{9}$ & $-0.13(0.03)$ & $0.03(0.03)$ \\
$d_{10}$ & $0.03(0.03)$ & $0.21(0.02)$ \\
$d_{11}$ & $-0.10(0.02)$ & $-0.18(0.02)$ \\
$d_{12}$ & $-0.43(0.05)$ & $-0.22(0.05)$ \\
$d_{13}$ & $-0.04(0.04)$ & $0.17(0.03)$ \\
$d_{14}$ & $-0.23(0.04)$ & $-0.22(0.03)$ \\
$d_{15}$ & $0.01(0.03)$ & $0.16(0.03)$ \\
$\delta$ & & $0.01(0.01)$ \\
\hline$R^{2}$ & 0.48 & 0.62
\end{tabular}

Table 4: Parameter estimates, standard errors and diagnostics obtained from fitting Model A and Model B to the NHL data.

facilitate a comparison, we refer to figure 1 which plots the size of the effects for both the NBA and the NHL data.

Our first observation is that there is general agreement in the ordering of the effects between the two leagues. A difference is that the NHL effects are slightly more compressed; NHL teams are less affected by the state of the series and tend to play on a more even keel from game to game. Perhaps this is due to the lesser role of the home team advantage in the NHL.

Second, in both leagues, we observe that the psychological impact of the match situation tends to become more extreme as the series progresses. In other words, larger effects (both positive and negative) are more strongly associated with games 5, 6 and 7 of a series. This makes sense from the point of view that as a team gets closer to elimination in a series, they 
play more desperately. For only then are the outcomes truly important as the consequences of winning/losing are dire. Conversely, we do not see strong effects early in series where teams have played a single game (i.e. State $1(1-0)$ and State $4(0-1)$ ).

An exception to the general rule that teams play more desperately later in series occurs with teams that have capitulated (given up). This is observed in States 3 (3-0), 8 (0-2) and $12(0-3)$. The "giving up" is not as strong in State 8 for the NHL compared to the NBA. This may again be explained by the lesser home team advantage in the NHL compared to the NBA. A team down 0-2 in the NBA has little chance as they are going on the road for two games. An NHL team is more hopeful.

A distinguishing feature between the NBA and the NHL is the that NBA teams bounce back after losses (negative $\delta$ in Model B) whereas a loss does not psychologically affect NHL teams ( $\delta \approx 0$ in Model $\mathrm{B}$ ). We have no physical explanation for this phenomenon but note that Stern (1998a) observed likewise in his analysis of an earlier dataset.

Finally, an irony in our analysis is that the entire discussion has been predicated on team psychology yet teams are composed of individuals. It is doubtful that all sports individuals behave the same way when confronted with the stresses imposed by the state of a playoff series. In particular, the disposition and performance of an NHL goaltender is crucial to his team's success. Perhaps one may find more variation in psychological performance in individual sports (e.g. tennis) than in team sports with a large number players (e.g. rugby union) where the individual effects may average out.

\section{REFERENCES}

Entine, O.A. and Small, D.S. (2008). "The role of rest in the NBA home-court advantage", Journal of Quantitative Analysis in Sports, 4(2), Article 6.

Gandar, J.M., Dare, W.H., Brown, C.R. and Zuber, R.A. (1998). "Informed traders and price variations in the betting market for professional basketball games", The Journal 
of Finance, 53(1), 385-401.

Gandar, J.M., Zuber, R.A. and Lamb, R.P. (2001). "The home field advantage revisted: a search for the bias in other sports betting markets", Journal of Economics and Business, 53(4), 439-453.

Gibbs, J. (2007). "An economic analysis of the National Basketball Association's point spread betting market", Undergraduate Honors Thesis, Department of Economics, Stanford University.

Jackson, D.A. (1993). "Independent trials are a model for disaster", Applied Statistics, 42, 211-220.

Jones, M.B. (2007). "Home advantage in the NBA as a game-long process", Journal of Quantitative Analysis in Sports, 3(4), Article 2.

Larsen, T., Price, J. and Wolfers. (2008). "Racial bias in the NBA: implications in betting markets", Journal of Quantitative Analysis in Sports, 4(2), Article 7.

Sarohia, P.S. (2010). "An analysis of the effect of desperation in sport", MSc dissertation, University of Victoria, Department of Mathematics and Statistics.

Sauer, R.D. (1998). "The economics of wagering markets", Journal of Economic Literature, 36(4), 2021-2064.

Schembri, A.J., Bedford, A., O'Bree, B. and Park, J.H. (2011). "Incentive versus expectation in NBA basketball", In Institute of Mathematics and its Applications, Third International Conference on Mathematics in Sport, Proceedings Papers (editors David Percy, J. James Read and Phil Scarf), The Lowry, Salford Quays, U.K., paper 33, 223-229. 
Stefani, R. (2008). "Measurement and interpretation of home advantage", In Statistical Thinking in Sports (editors J. Albert and R.H. Koning), Chapman \& Hall/CRC, 203216.

Stern, H.S. and Mock, B. (1998). "College basketball upsets: will a 16-seed ever beat a 1-seed?", In the column, "A Statistician Reads the Sports Pages", Chance 11(1), 26-31.

Stern, H.S. (1998a). "Best-of-seven playoff series", In the column, "A Statistician Reads the Sports Pages", Chance 11(2), 46-49.

Stern, H.S. (1998b). "How accurate are the posted odds?", In the column, "A Statistician Reads the Sports Pages", Chance 11(4), 17-21.

Tennakoon, A. (2011). "Desperation in Sport", MSc project, Simon Fraser University, Department of Statistics and Actuarial Science.

Tversky, A. and Gilovich, T. (1989), "The cold facts about the 'hot hand' in basketball". Chance 2(1), 16-21. 


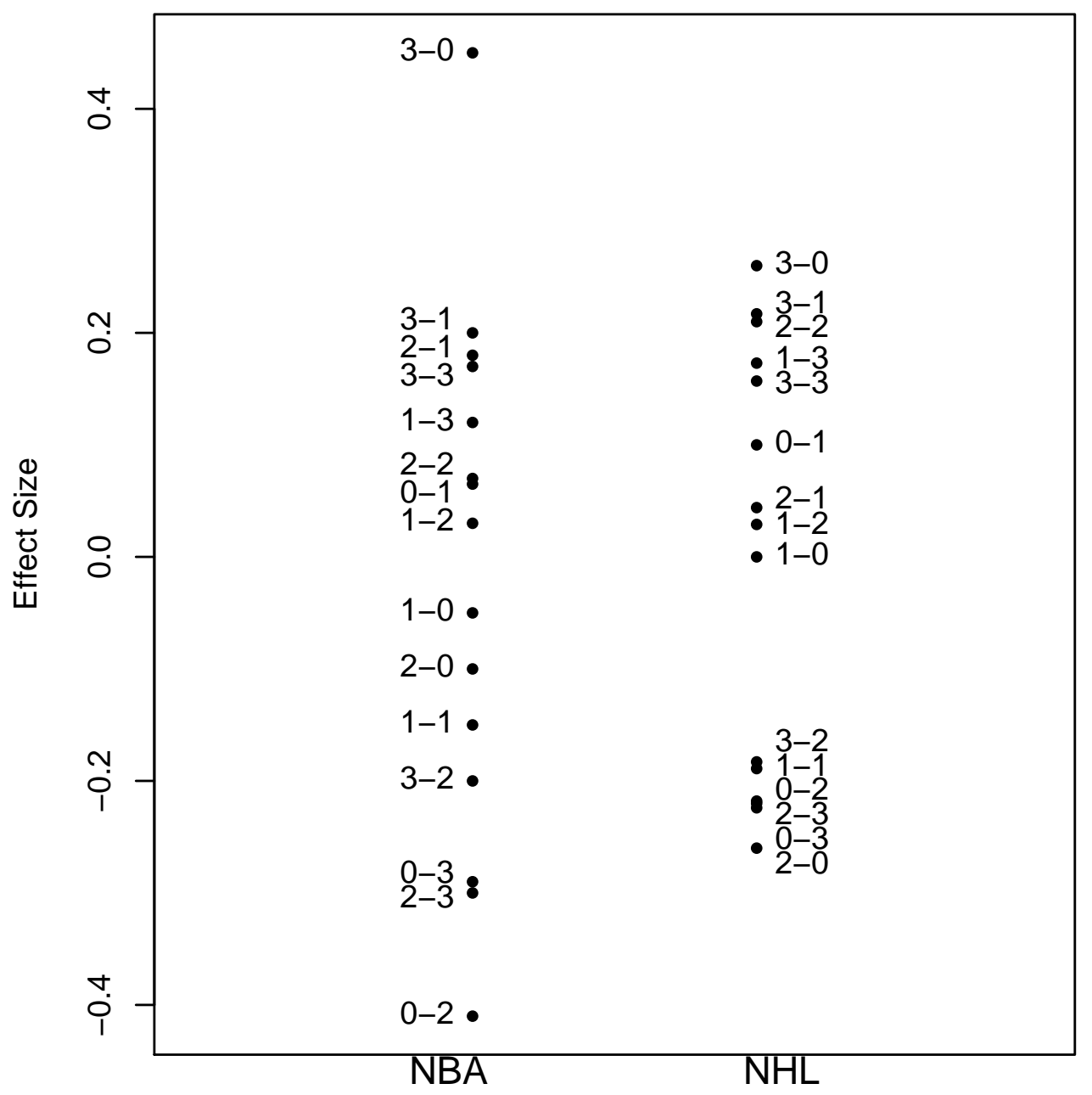

Figure 1: Estimates of the NBA and NHL parameters $d_{j}$ corresponding to Model B. 